JOURNAL OF THE

AMERICAN MATHEMATICAL SOCIETY

Volume 10, Number 2, April 1997, Pages 427-443

S 0894-0347(97)00230-0

\title{
MAXIMAL DEGENERACY POINTS OF GKZ SYSTEMS
}

\author{
S. HOSONO, B. H. LIAN, AND S.-T. YAU
}

\section{INTRODUCTION}

In this paper, we study the Gel'fand-Kapranov-Zelevinsky hypergeometric PDE systems [12]. We are mainly concerned with a case of the so-called resonant exponent $\beta$, though some of our results apply to generic $\beta$ as well. This resonant case in fact plays an especially important role for applications in mirror symmetry.

The problem we consider is motivated by mirror symmetry [25] as follows. Given a family of Calabi-Yau manifolds $\pi: \mathcal{X} \rightarrow S$, one would like to give a suitable compactification in which there is a boundary point, called a large radius limit, where all but one of period integrals of the manifolds become singular near that point. An astonishing discovery [6] in mirror symmetry is that, under some assumptions, the period integrals near this point combine to form a generating function which predicts the number of rational curves (a.k.a. instanton contributions) on another Calabi-Yau manifold. Thus the existence and construction of a large radius limit pose an important problem.

When the Calabi-Yau manifolds are anticanonical divisors in a suitable toric variety, then their period integrals are natural solutions to a GKZ system [2]. Thus the GKZ theory becomes an important tool for studying the behavior of such period integrals ([15], [16], [4], [17]). Motivated by this, we study a GKZ system canonically associated with a complete regular fan, and a class of integral solutions. We prove a general existence theorem for the so-called maximal degeneracy points [19] for these integral representations. These special points can be geometrically interpreted as fixed points under a canonical torus action on the parameter space. Some complete regular fans (in most interesting examples we know) arise in mirror symmetry [1]. When they do, the maximal degeneracy points turn out to be large radius limits where the enumeration of instanton contributions is carried out ([6], [19], [18], [10], [7], [15], [8], [16], [4], [17]).

\subsection{Conventions and notation.}

$$
\begin{aligned}
N & : \text { rank } n \text { lattice } \\
M & : \text { dual lattice } \\
N_{\mathbf{R}} & : N \otimes \mathbf{R} \\
M_{\mathbf{R}} & : M \otimes \mathbf{R} \\
\bar{N} & : \mathbf{Z} \times N \\
\bar{M} & : \mathbf{Z} \times M
\end{aligned}
$$

Received by the editors May 28, 1996 and, in revised form, November 13, 1996.

1991 Mathematics Subject Classification. Primary 14C30, 32G20.

Key words and phrases. Mirror symmetry, hypergeometric systems, period integrals, CalabiYau manifolds, toric varieties, compactification, indicial ideal, Gröbner bases. 
$\mu:$ a point in $N$

$\bar{\mu}: 1 \times \mu$

$\Sigma$ : a complete rational polyhedral fan in $N$

$\mathcal{A}$ : a finite set of integral points which spans the affine hyperplane $1 \times N_{\mathbf{R}} \subset$ $\bar{N}_{\mathbf{R}}$

$L=L_{\mathcal{A}}$ : the lattice of relations on $\mathcal{A}$

$\mathbf{P}_{\Sigma}$ : the toric variety associated to the fan $\Sigma$

$\Sigma(1)$ : the set of primitive elements on the edges of the fan $\Sigma$

$\sigma(1)$ : the set of primitive elements on the edges of the cone $\sigma$

$S \Sigma$ : the secondary fan of $\Sigma$

$G \Sigma$ : the Gröbner fan

cone $(S)$ : the cone generated by a finite set $S$

$\operatorname{conv}(S)$ : the convex hull of a finite set $S$

An $n$-dimensional cone in $N_{\mathbf{R}}$ is also called a large cone. A cone which contains no nontrivial linear subspace is called strongly convex. A cone generated by finitely many integral points is called rational. A rational cone is called regular if it is generated by a subset of an integral base. A fan is regular if it consists of regular cones. The dual of a cone $\sigma$ is denoted $\sigma^{\vee}$.

\section{GKZ $\mathcal{A}$-hypergeometric Systems}

Let $\mathcal{A} \subset \bar{N}$ be a set of $p+1$ integral points which span the affine plane $1 \times N_{\mathbf{R}}$. We sometimes write $\mathcal{A}=\left\{\bar{\mu}_{0}, \ldots, \bar{\mu}_{p}\right\}$. The projection map $\bar{N} \rightarrow N$ is denoted $\bar{\mu} \mapsto \mu$. Let $L=L_{\mathcal{A}}:=\left\{l \in \mathbf{Z}^{p+1} \mid \sum_{\bar{\mu} \in \mathcal{A}} l_{\mu} \bar{\mu}=0\right\}$, which we call the lattice of relations on $\mathcal{A}$. This is clearly a lattice of rank $p-n$. The GKZ system with exponent $\beta \in \bar{N}_{\mathbf{R}}$ is the PDE system on $\mathbf{C}^{p+1}=\mathbf{C}^{\mathcal{A}}$ given by

$$
\square_{l} \Pi(a)=0, \quad\left(\sum\langle u, \bar{\mu}\rangle \theta_{a_{\mu}}-\langle u, \beta\rangle\right) \Pi(a)=0
$$

for $l \in L, u \in \bar{M}_{\mathbf{R}}[12]$. Here $\square_{l}:=\prod_{l_{\mu}>0}\left(\frac{\partial}{\partial a_{\mu}}\right)^{l_{\mu}}-\prod_{l_{\mu}<0}\left(\frac{\partial}{\partial a_{\mu}}\right)^{-l_{\mu}}$ and $\theta_{a_{\mu}}:=$ $a_{\mu} \frac{\partial}{\partial a_{\mu}}$. Equivalently we can write

$$
a^{-\gamma} \square_{l} a^{\gamma} f(a)=0, \quad \sum\langle u, \bar{\mu}\rangle \theta_{a_{\mu}} f(a)=0,
$$

where $f(a)=a^{-\gamma} \Pi(a)$ and $\gamma$ lies in the $(p-n)$-dimensional affine subspace $\Phi(\beta):=$ $\left\{\gamma \mid \sum_{\mu} \gamma_{\mu} \bar{\mu}=\beta\right\}$. We shall always assume that $\bar{\mu}_{0}:=1 \times \overrightarrow{0} \in \mathcal{A}$.

\section{Period integrals}

We shall consider a class of (multi-valued) analytic functions attached to the data $\mathcal{A}$ as follows. Given a choice of basis $e_{1}, \ldots, e_{n}$ of $N$, we have an isomorphism $T_{M}=\operatorname{Hom}\left(N, \mathbf{C}^{\times}\right) \cong\left(\mathbf{C}^{\times}\right)^{n}$. On this algebraic torus, there is a family of rational functions defined by the Laurent polynomials:

$$
f_{\mathcal{A}}(X, a)=\sum_{\bar{\mu} \in \mathcal{A}} a_{\mu} X^{\mu} .
$$

The zero locus of this function is a divisor $H_{a}$ in the torus $T_{M}$. Now $T_{M}$ has a canonical $T_{M}$-invariant holomorphic $n$-form given by $\prod \frac{d X_{i}}{X_{i}}$. Following [2], for every homology $n$-cycle $\alpha$ in the manifold $T_{M}-H_{a}$, we define the period integral

$$
\Pi_{\alpha}(a)=\int_{\alpha} \frac{1}{f_{\mathcal{A}}(X, a)} \prod \frac{d X_{i}}{X_{i}} .
$$


This integral converges in some domain, and is singular in some divisor $D \subset \mathbf{C}^{\mathcal{A}}$. Under analytic continuation in $\mathbf{C}^{\mathcal{A}}-D$, the integral will in general have monodromy. Thus the period integrals are local sections of a locally constant sheaf over $\mathbf{C}^{\mathcal{A}}-D$. As an example, we have the following. Let $\alpha_{0}$ be the real torus cycle $\left\{\left|X_{1}\right|=\cdots=\right.$ $\left.\left|X_{n}\right|=1\right\}$. The following two results are proved in [2].

Proposition 3.1. In the domain $\left|a_{0}\right| \gg\left|a_{1}\right|, \ldots,\left|a_{p}\right|$, we have the convergent power series representation

$$
\begin{aligned}
& a_{0} \int_{\alpha_{0}} \frac{1}{f_{\mathcal{A}}(X, a)} \prod_{i=1}^{n} \frac{d X_{i}}{X_{i}} \\
& \quad=(2 \pi i)^{n} \sum_{l_{1} \mu_{1}+\cdots+l_{p} \mu_{p}=0, l_{1}, \ldots, l_{p} \geq 0}(-1)^{l_{1}+\cdots+l_{p}} \frac{\left(l_{1}+\cdots+l_{p}\right) !}{l_{1} ! \cdots l_{p} !} \frac{a_{1}^{l_{1}} \cdots a_{p}^{l_{p}}}{a_{0}^{l_{1}+\cdots+l_{p}}} .
\end{aligned}
$$

Proof. First we have the geometric series

$$
\frac{a_{0}}{f_{\mathcal{A}}(X, a)}=\sum_{r \geq 0} \frac{1}{a_{0}^{r}}\left(-a_{1} X^{\mu_{1}}-\cdots-a_{p} X^{\mu_{p}}\right)^{r} .
$$

Also we have

$$
\int_{\alpha_{0}} X^{\lambda} \prod_{i=1}^{n} \frac{d X_{i}}{X_{i}}=(2 \pi i)^{n} \delta_{\lambda, 0} .
$$

Thus the terms in the geometric series which contribute to the integral are powers of the form $(-1)^{l_{1}+\cdots+l_{p}} \frac{a_{1}^{l_{1}} \ldots a_{p}^{l_{p}}}{a_{0}^{l_{1}+\cdots+l_{p}}}$, where $l_{1} \mu_{1}+\cdots+l_{p} \mu_{p}=0, l_{1}, \ldots, l_{p} \geq 0$. In each term $\frac{1}{a_{0}^{r}}\left(-a_{1} X^{\mu_{1}}-\cdots-a_{p} X^{\mu_{p}}\right)^{r}$, each tuple $\left(l_{1}, \ldots, l_{p}\right)$ with $r=l_{1}+\cdots+l_{p}$ contributes exactly $\frac{\left(l_{1}+\cdots+l_{p}\right) !}{l_{1} ! \cdots l_{p} !}$ copies of such a power. Now summing over all $r \geq 0$ and over all those tuples, we get the desired sum. The sum is convergent because it is a subseries of the standard series (with $z_{i}=-a_{i} / a_{0}$ ):

$$
\sum_{l_{1}, \ldots, l_{p} \geq 0} \frac{\left(l_{1}+\cdots+l_{p}\right) !}{l_{1} ! \cdots l_{p} !} z_{1}^{l_{1}} \cdots z_{p}^{l_{p}} .
$$

Proposition 3.2 (see [13]). The period integrals $\Pi_{\alpha}(a)$ are solutions to the $G K Z$ system with the exponent $\beta=-1 \times \overrightarrow{0}$.

Proof. We claim that $\square_{l} \Pi_{\alpha}(a)=0$ for all $l \in L$. In fact, $\square_{l} G\left(f_{\mathcal{A}}(a, X)\right)=0$ for any differentiable function $G$. First we have

$$
\partial_{a_{j_{1}}} \cdots \partial_{a_{j_{k}}} G\left(f_{\mathcal{A}}(a, X)\right)=G^{(k)}(f) X^{\mu_{j_{1}}} \cdots X^{\mu_{j_{k}}} .
$$

Write $l \in L$ as $l=l^{+}-l^{-}$, where $l^{ \pm}$have only nonnegative entries and have disjoint support. Note that the sum of the entries of each $l^{+}$is the same as that of $l^{-}$. Call this $\operatorname{sum} k$. Then we have

$$
\square_{l} G\left(f_{\mathcal{A}}(a, X)\right)=G^{(k)}\left(f_{\mathcal{A}}\right)\left(X^{\sum l_{\mu}^{+} \mu}-X^{\sum l_{\mu}^{-} \mu}\right) .
$$

But $l \in L$ implies that $0=\sum l_{\mu} \mu=\sum\left(l_{\mu}^{+}-l_{\mu}^{-}\right) \mu$. It follows that $\square_{l} G\left(f_{\mathcal{A}}(a, X)\right)=$ 0 .

Now we have to check that the periods $\Pi_{\alpha}(a)$ are annihilated by the order 1 operators in the GKZ system. We consider the action of $\lambda:=\left(\lambda_{0}, \lambda_{1}, \ldots, \lambda_{n}\right)$ 
on the periods as follows: under $X_{i} \mapsto \lambda_{i} X_{i}$, the cycle $\alpha$ gets moved to $\alpha_{\lambda}$ and $d m:=\prod_{i=1}^{n} \frac{d X_{i}}{X_{i}}$ is invariant. Thus we get

$$
\frac{1}{\lambda_{0}} \int_{\alpha} \frac{1}{\sum a_{\mu} X^{\mu}} d m=\int_{\alpha_{\lambda}} \frac{1}{\sum a_{\mu} \lambda^{\bar{\mu}} X^{\mu}} d m .
$$

Now for $\lambda$ closed enough to the identity element, it is clear that the cycle $\alpha_{\lambda}$ is homologous to $\alpha$. So we can replace $\alpha_{\lambda}$ on the RHS by $\alpha$. Differentiating the equation above by $\lambda_{i} \frac{\partial}{\partial \lambda_{i}}$ and then setting $\lambda=\mathrm{id}=(1, \ldots, 1)$, we get

$$
\begin{aligned}
-\left.\frac{1}{\lambda_{0}}\right|_{\lambda=\mathrm{id}} \delta_{i, 0} \int_{\alpha} \frac{1}{\sum a_{\mu} X^{\mu}} d m & =\left.\int_{\alpha} \frac{-\sum a_{\mu} \bar{\mu}_{i} \lambda^{\bar{\mu}} X^{\mu}}{\left(\sum a_{\mu} \lambda^{\bar{\mu}} X^{\mu}\right)^{2}} d m\right|_{\lambda=\mathrm{id}} \\
& =\int_{\alpha} \frac{-\sum\left\langle e_{i}, \bar{\mu}\right\rangle a_{\mu} X^{\mu}}{f_{\mathcal{A}}(a, X)^{2}} d m \\
& =\sum\left\langle e_{i}, \bar{\mu}\right\rangle \theta_{a_{\mu}} \int_{\alpha} \frac{1}{f_{\mathcal{A}}(a, X)} d m
\end{aligned}
$$

where $\theta_{a_{\mu}}:=a_{\mu} \frac{\partial}{\partial a_{\mu}}$. It follows that $\left\langle e_{i}, \beta\right\rangle \Pi_{\alpha}(a)=\sum_{\mu}\left\langle e_{i}, \bar{\mu}\right\rangle \theta_{a_{\mu}} \Pi_{\alpha}(a)$.

Remark 3.3. Note that the order 1 operators in the GKZ system are given by the vector field of the algebraic group $T_{M}$ with some twist given by the exponent $\beta=-1 \times \overrightarrow{0}$. Thus the order 1 equations mean that the period integrals are of the form $a_{0}^{-1}$ times a function invariant under $\mathbf{C}^{\times} \times T_{M}$ which acts by $\lambda:\left(a_{\mu}\right) \mapsto\left(a_{\mu} \lambda^{\bar{\mu}}\right)$, where $\lambda=\left(\lambda_{0}, \ldots, \lambda_{n}\right)$.

3.1. The maximal degeneracy problem. Since the GKZ system is defined on a domain in $\mathbf{C}^{p+1}=\mathbf{C}^{\mathcal{A}}$, it is natural to study how the solutions degenerate near the "boundary" of the domain. This should tell us something about how the period integrals of the hypersurfaces $f_{\mathcal{A}}=0$ degenerate. This of course depends on how we compactify the domain. Now any nonzero $f_{\mathcal{A}}$ defines a hypersurface. Thus it is natural to consider the projective space $\mathbf{P} \mathbf{C}^{\mathcal{A}}$. As remarked in 3.3 the $\mathrm{GKZ}$ system has a symmetry group given by the algebraic torus $\mathbf{C}^{\times} \times T_{M} \cong\left(\mathbf{C}^{\times}\right)^{n+1}$. It seems natural to consider the quotient $\mathbf{P C}^{\mathcal{A}} /\left(\mathbf{C}^{\times} \times T_{M}\right)$. If $G$ is an algebraic group acting on a variety $V, V / G$ in general will not be a variety. However, note that $\mathbf{P} \mathbf{C}^{\mathcal{A}}$ admits a canonical action by $\left(\mathbf{C}^{\times}\right)^{\mathcal{A}}$, and the group $\mathbf{C}^{\times} \times T_{M}$ acts by a subgroup. Thus we expect " $\mathbf{P} \mathbf{C}^{\mathcal{A}} /\left(\mathbf{C}^{\times} \times T_{M}\right)$ " to be a toric variety with a $(p-n)$ dimensional open torus. There are two ways to repair the quotient. We can use geometric invariant theory [20] to construct a model for the quotient. We shall, however, use a different approach which we now explain. A virtue of this approach is that the resulting compactification can be described quite explicitly.

Proposition 3.4. The map $\left(\mathbf{C}^{\times}\right)^{\mathcal{A}} /\left(\mathbf{C}^{\times} \times T_{M}\right) \rightarrow \operatorname{Hom}\left(L, \mathbf{C}^{\times}\right)$given by $\left(a_{\mu}\right) \mapsto$ $x_{a}$, where $x_{a}(l)=(-1)^{l_{0}} a^{l}$, is an isomorphism.

The extra sign is only a matter of convenience here, but is important in applications to mirror symmetry in physics [15]. We denote by $S_{\mathcal{A}}$ the image of $\left(\mathbf{C}^{\times}\right)^{\mathcal{A}}-D$ under the above isomorphism. This is the complement of a divisor in $\operatorname{Hom}\left(L, \mathbf{C}^{\times}\right)$. By the standard construction of toric varieties from fans, we see that any toric variety $\mathbf{P}_{F}$ associate with a complete fan $F$ in $L_{\mathbf{R}}^{*}$ is a compactification of $\operatorname{Hom}\left(L, \mathbf{C}^{\times}\right)$ and hence of $S_{\mathcal{A}}$. Since the period integrals $\bar{\Pi}_{\alpha}:=a_{0} \Pi_{\alpha}(a)$ are $\mathbf{C}^{\times} \times T_{M}$-invariant, they descend to local sections of a locally constant sheaf over $S_{\mathcal{A}}$. 
Definition 3.5. We call a smooth boundary point $p \in \mathbf{P}_{F}-\operatorname{Hom}\left(L, \mathbf{C}^{\times}\right)$a maximal degeneracy point if near $p$ there is exactly one period integral (up to a multiple) $\bar{\Pi}_{\alpha}$ which extends holomorphically across $p$.

We will find in Section 6 a natural compactification $\mathbf{P}_{F}$-one which can be described in terms of the initial data $\mathcal{A}$ alone - and we will prove the existence of maximal degeneracy points in this compactification.

\section{The SECONDARY FAN AND GRÖBNER FAN}

4.1. The secondary fan. Let us consider the convex polytope $\operatorname{conv}(\mathcal{A})$. A triangulation of $\operatorname{conv}(\mathcal{A})$ (see [12]) is a collection of $n$-simplices whose vertices are in $\mathcal{A}$, such that the intersection of two such simplices is a face of both. Thus $T$ is given by a collection of bases $I \subset \mathcal{A}$ of $\bar{N}_{\mathbf{R}}$, each basis being the set of vertices of an $n$-simplex. A continuous function $h$ on the cone over $\operatorname{conv}(\mathcal{A}) \subset \bar{N}_{\mathbf{R}}$ is $T$ piecewise linear if it is linear in the cone over each $n$-simplex of $T$. Note that $h$ is determined by its values at the vertices $\bar{\mu}$ of $n$-simplices in $T$. Thus each point in $x \in \mathbf{R}^{p+1}=\mathbf{R}^{\mathcal{A}}$ determines a piecewise linear function $h_{x}$ whose values at a vertex $\bar{\mu}_{i}$ is $x_{i}$. A piecewise linear function $h_{x}$ is called convex if $h_{x}(a+b) \leq h_{x}(a)+h_{x}(b)$ for arbitrary $a, b$ in the cones, and is called strictly convex if in addition it satisfies $\left.h_{x}\right|_{\sigma} \neq\left. h_{x}\right|_{\tau}$ for any large cones $\sigma, \tau$.

Let $\mathcal{C}^{\prime}(T)$ be the set of points $x$ for which $h_{x}$ is convex and has the property that $h_{x}\left(\bar{\mu}_{i}\right) \leq x_{i}$ if $\bar{\mu}_{i}$ is not a vertex of $T$. It is known that $\mathcal{C}^{\prime}(T)$ is a polyhedral cone in $\mathbf{R}^{p+1}$ but it is not strongly convex. The triangulation $T$ is called regular if $\mathcal{C}^{\prime}(T)$ has an interior point, i.e., there is a strictly convex $T$-piecewise linear function. Define for each basis $I$ of $N_{\mathbf{R}}$,

$$
\mathcal{C}^{\prime}(I):=\left\{x \in \mathbf{R}^{p+1} \mid h_{I, x}\left(\bar{\mu}_{j}\right) \leq x_{j}, \forall j \notin I\right\},
$$

where $h_{I, x}$ is the linear function with value $x_{i}$ at $\bar{\mu}_{i}, i \in I$. We also define for the basis $I$ a subspace of $L_{\mathbf{R}}$,

$$
K(I):=\left\{x \in L_{R} \mid x_{i} \geq 0, \forall i \notin I\right\} .
$$

Then

Proposition 4.1 ([12]). If $I \subset \mathcal{A}$ is a basis of $\bar{N}_{\mathbf{R}}$, then $\mathcal{C}^{\prime}(I)^{\vee}=K(I)$. If $T$ is a triangulation of $\operatorname{conv}(\mathcal{A})$, then $\mathcal{C}^{\prime}(T)=\bigcap_{I \in T} \mathcal{C}^{\prime}(I)$.

The collection of the cones $\mathcal{C}^{\prime}(T)$ with $T$ regular, together with their faces form a fan called the secondary fan $S \Sigma$. Now every cone contains a minimal face which is a linear space of dimension $n+1$. This is exactly the space of points $x$ for which $\left\{\left.h_{x}\right|_{\sigma}\right\}$ glues to a global linear function. This space may be naturally identified with $\bar{N}_{\mathbf{R}}^{*}=\bar{M}_{\mathbf{R}}$. If we project the secondary fan in $\mathbf{R}^{p+1}$ along the $\bar{M}_{\mathbf{R}}$, we obtain a new fan in $\mathbf{R}^{p+1} / \bar{M}_{\mathbf{R}}$. We also call this the secondary fan. It is known that the secondary fan now consists of strongly convex cones and is a complete fan. The image of the cone $\mathcal{C}^{\prime}(T)$ under the projection will be denoted $\mathcal{C}(T)$. Again under the natural identification $\mathbf{R}^{p+1} / \bar{M}_{\mathbf{R}}=L_{\mathbf{R}}^{*}$, we can regard each cone $\mathcal{C}(T)$, hence the whole secondary fan, as lying in $L_{\mathbf{R}}^{*}$.

4.2. Toric ideal and the Gröbner fan. We briefly review some results of Sturmfels [23], [24]. Consider the generic orbit $T_{M} \cdot[1, \ldots, 1]$ in $\mathbf{P C}^{\mathcal{A}}$. The closure of this orbit is a projective toric variety. Its vanishing ideal $\mathcal{I}_{\mathcal{A}}$ is a homogeneous prime 
ideal called a toric ideal, in $\mathbf{C}\left[y_{0}, \ldots, y_{p}\right]$. It is known that $\mathcal{I}_{\mathcal{A}}=\left\langle y^{l^{+}}-y^{l^{-}} \mid l \in L\right\rangle$. Here $l_{\mu}^{+}=l_{\mu}$ if $l_{\mu} \geq 0$ and zero otherwise, and similarly for $l^{-}$.

Every vector $\omega \in \mathbf{R}^{p+1}=\mathbf{R}^{\mathcal{A}}$ induces a polyhedral subdivision $T_{\omega}$ of $\mathcal{A}$ as follows. Consider the $(n+1)$-polytope $P_{\omega}:=\operatorname{conv}\left(\left\{\left(\omega_{\mu}, \mu\right) \mid \bar{\mu} \in \mathcal{A}\right\}\right)$ which is a lifting of $\operatorname{conv}(\mathcal{A})=\operatorname{conv}(\{(1, \mu) \mid \bar{\mu} \in \mathcal{A}\})$ according to the height vector $\omega$. The lower envelope of $P_{\omega}$ is a polyhedral $n$-ball which maps bijectively onto $\operatorname{conv}(\mathcal{A})$. Let $T_{\omega}$ be the image under this projection. If $\omega$ is sufficiently generic, $T_{\omega}$ is a triangulation of $\mathcal{A}$ (really of $\operatorname{conv}(\mathcal{A})$ ). A triangulation $T$ of $\mathcal{A}$ is regular iff $T=T_{\omega}$ for some $\omega$. When $T$ is regular, the set of all $\omega$ with $T=T_{\omega}$ is the $\operatorname{interior} \operatorname{Int}\left(\mathcal{C}^{\prime}(T)\right)$ of the cone $\mathcal{C}^{\prime}(T)$ in the secondary fan.

Given a vector $\omega$, we define a weight on the set of monomials in $y_{0}, \ldots, y_{p}$, by $t_{\omega}\left(y^{l}\right)=w_{0} l_{0}+\cdots+w_{p} l_{p}$. Consider now the leading term ideal $L T_{\omega}\left(\mathcal{I}_{\mathcal{A}}\right)$ of the toric ideal $\mathcal{I}_{\mathcal{A}}$, relative to the weight $t_{\omega}$. Two vectors $\omega, \omega^{\prime}$ are said to be equivalent if $L T_{\omega}\left(\mathcal{I}_{\mathcal{A}}\right)=L T_{\omega^{\prime}}\left(\mathcal{I}_{\mathcal{A}}\right)$. The equivalence classes of such vectors form a complete fan in $\mathbf{R}^{p+1}$. Projecting this fan along the linear subspace $\bar{M}_{\mathbf{R}} \hookrightarrow \mathbf{R}^{p+1}$ (given by $u \mapsto \sum\left\langle u, \bar{\mu}_{i}\right\rangle e_{i} \in \mathbf{R}^{p+1}$ ), we get a fan in $L_{\mathbf{R}}^{*}=\mathbf{R}^{p+1} / \bar{M}_{R}$, which is called the Grob̈ner fan $G \Sigma$ for the toric ideal $\mathcal{I}_{\mathcal{A}}$. An interior point of a large cone in $G \Sigma$ (or its lifting in $\mathbf{R}^{p+1}$ ) is called a term order for $\mathcal{I}_{\mathcal{A}}$.

The Stanley-Reisner ideal $S R_{T}$ for a triangulation $T$ of $\mathcal{A}$ is the ideal in $\mathbf{C}\left[y_{0}, \ldots, y_{p}\right]$ generated by all monomials $y_{I}:=y_{i_{1}} \cdots y_{i_{k}}$ where $I=\left\{\bar{\mu}_{i_{1}}, \ldots, \bar{\mu}_{i_{k}}\right\} \subset$ $\mathcal{A}$ is not a simplex of $T$. A collection of vertices $\mathcal{P}=\left\{\bar{\mu}_{i_{1}}, \ldots, \bar{\mu}_{i_{k}}\right\}$ is called primitive if $\mathcal{P}$ is not a simplex contained in $T$ but any subset of it is a simplex. Then it is easy to see that $S R_{T}$ is generated by the $y_{\mathcal{P}}$ with $\mathcal{P}$ being a primitive collection (see below) of $T$.

Theorem $4.2([23])$. Let $\omega$ be a term order of the toric ideal $\mathcal{I}_{\mathcal{A}}$. Then the polyhedral subdivision $T_{\omega}$ is a triangulation of $\mathcal{A}$. Moreover, $S R_{T_{\omega}}=\operatorname{rad} L T_{\omega}\left(\mathcal{I}_{\mathcal{A}}\right)$ and $G \Sigma$ is a refinement of $S \Sigma$.

4.3. Toric variety $\mathbf{P}_{\Sigma}$ and Chow ring. Let us consider a complete fan $\Sigma$ in $N$ with the property that the set of the primitive generators $\Sigma(1)$ is given by

$$
\Sigma(1)=\{\mu \mid \bar{\mu} \in \mathcal{A},(\mu \neq 0)\} .
$$

The fan $\Sigma$ need not be regular in general. In the following sections, we will restrict our attention to the case in which $\Sigma$ is regular. In this case, the toric variety associated to the fan $\Sigma$ is a complete smooth toric variety $\mathbf{P}_{\Sigma}$.

Theorem 4.3 (see [21], [11]). For a complete smooth toric variety $\mathbf{P}_{\Sigma}$, the intersection ring $A^{*}\left(\mathbf{P}_{\Sigma}\right)$ is given by $\mathbf{Z}\left[D_{1}, \ldots, D_{p}\right] / \mathcal{I}$, where $\mathcal{I}$ is the ideal generated by

(i) $D_{i_{1}} \cdots D_{i_{k}}$ for $\mu_{i_{1}}, \ldots, \mu_{i_{k}}$ not in a cone of $\Sigma$,

(ii) $\sum_{i=1}^{p}\left\langle u, \mu_{i}\right\rangle D_{i}$ for $u \in M$.

We call $\mathcal{I}$ the Chow ideal of $\mathbf{P}_{\Sigma}$. Note the similarity of (i) to the Stanley-Reisner ideal for a triangulation of $\mathcal{A}$. Because of this we call the ideal generated by (i) the Stanley-Reisner ideal of $\Sigma$. Equivalently, this is the ideal generated by the elements $D_{i_{1}} \cdots D_{i_{k}}$ with $\left\{\mu_{i_{1}}, \ldots, \mu_{i_{k}}\right\}$ ranging over all primitive collections of the fan $\Sigma$ (see below for the definition). It follows from the theorem that $A^{1}\left(\mathbf{P}_{\Sigma}\right)=$ $H^{2}\left(\mathbf{P}_{\Sigma}, \mathbf{Z}\right)=\mathbf{Z}^{p} / M$. 
Definition 4.4 (see [3]). A primitive collection of a complete fan $\Sigma$ is a subset $\mathbf{P} \subset \Sigma(1)$ that does not generate a cone in $\Sigma$, but $\mathcal{P}-\mu$ generates a cone in $\sigma$ for every $\mu \in \mathcal{P}$.

Consider a piecewise linear function relative to $\Sigma$. As before, the set of all piecewise linear functions $P L(\Sigma)$ is canonically isomorphic to $\mathbf{R}^{p}=\mathbf{R}^{\Sigma(1)}$. The subset of the strictly convex piecewise linear functions will be denoted as $K\left(\mathbf{P}_{\Sigma}\right)$. Under our assumption of regularity of the fan $\Sigma$, we have

Theorem 4.5 (see [21]). $P L(\Sigma) / M_{\mathbf{R}} \cong H^{2}\left(\mathbf{P}_{\Sigma}, \mathbf{R}\right)$, and $K\left(\mathbf{P}_{\Sigma}\right)$ is the Kähler cone of $\mathbf{P}_{\Sigma}$. Moreover, the first Chern class $c_{1}\left(\mathbf{P}_{\Sigma}\right):=c_{1}\left(\Theta_{\mathbf{P}_{\Sigma}}\right)$ is represented by $\alpha_{\Sigma} \in P L(\Sigma)$ with $\alpha(\mu)=1$ for all $\mu \in \Sigma(1)$.

Theorem 4.6 (see [3]). $f \in K\left(\mathbf{P}_{\Sigma}\right)$ iff $\sum_{\mu \in \mathcal{P}} f(\mu)>f\left(\sum_{\mu \in \mathcal{P}} \mu\right)$ for all primitive collections $\mathcal{P}$.

Let $\mathcal{P} \subset \Sigma(1)$ be a primitive collection. Then there is a unique cone $\sigma \in \Sigma$ of minimal dimension such that $\sum_{\mu \in \mathcal{P}} \mu \in \operatorname{Int}(\sigma)$. By regularity $\sigma$ has a unique set of integral generators $\mathcal{G} \subset \Sigma(1)$. Thus we have a relation

$$
\sum_{\mu \in \mathcal{P}} \mu=\sum_{\mu \in \mathcal{G}} c_{\mu} \mu
$$

for some positive integral $c_{\mu}$ (positive because the LHS is in the interior; integral because LHS is a lattice point). Equivalently we can write

$$
\sum_{\mu \in \mathcal{P}} \bar{\mu}=c_{0} \bar{\mu}_{0}+\sum_{\mu \in \mathcal{G}} c_{\mu} \bar{\mu}
$$

where $\bar{\mu}_{0}=1 \times \overrightarrow{0}$ and $c_{0}:=|\mathcal{P}|-\sum_{\mu \in \mathcal{G}} c_{\mu}$.

Proposition 4.7. The sets $\mathcal{P}, \mathcal{G} \cup\{\overrightarrow{0}\}$ are disjoint. Hence (4.5) defines an element $l(\mathcal{P}) \in L$, which is called a primitive relation. If $l(\mathcal{P})_{0} \leq 0$, then $l(\mathcal{P})^{ \pm}$are given by the left- and right-hand sides of (4.5), respectively.

Proof. First note that the fact that $\mathcal{P}$ is primitive implies that $\overrightarrow{0} \notin \mathcal{P}$. Thus we need to show that $\mathcal{P}, \mathcal{G}$ are disjoint. Suppose $\lambda$ is in both. Then we have

$$
\sum_{\mu \in \mathcal{P}-\lambda} \mu=\sum_{\mu \in \mathcal{G}-\lambda} c_{\mu} \mu+\left(c_{\lambda}-1\right) \lambda .
$$

But $\mathcal{P}-\lambda$ generates a cone $\tau$ and so the LHS of (4.6) is in $\operatorname{Int}(\tau)$. But we know the RHS is in $\sigma$. This means that both sides are in $\tau \cap \sigma$. So both sides are either in $\partial \tau$ or in $\partial \sigma$ or else $\tau=\sigma$. It cannot be in $\partial \tau$ because it is in $\operatorname{Int}(\tau)$. We cannot have $\tau=\sigma$ because this would mean that $\mathcal{P}-\lambda=\mathcal{G}$ since these are their respective generating sets. This would contradict $\lambda \in \mathcal{G}$.

So finally suppose both sides are in $\partial \sigma$. This means that $\tau \subset \partial \sigma$. This implies on the RHS of (4.6) one of the coefficients of those generators must be zero. Now $c_{\mu}>0$ means that $c_{\lambda}-1=0$. Since the cones $\tau, \sigma$ are regular, they have unique sets of generators. In particular, the generators of $\tau$ must be a subset of generators of $\sigma$, i.e. $\mathcal{P}-\lambda \subset \mathcal{G}$. But since both sides of (4.6) have a unique nonnegative decomposition in terms of $\mathcal{G}$, it follows that $\mathcal{P}-\lambda=\mathcal{G}-\lambda$. Hence $\mathcal{P}=\mathcal{G}$, which contradicts that $\mathcal{P}$ is primitive.

Proposition 4.8. $\bar{K}\left(\mathbf{P}_{\Sigma}\right)^{\vee}$ is generated by the primitive relations. 
Proof. The natural pairing between $H_{2}\left(\mathbf{P}_{\Sigma}, \mathbf{R}\right)=L_{\mathbf{R}}$ and $H^{2}\left(\mathbf{P}_{\Sigma}, \mathbf{R}\right)=P L(\Sigma) / M_{\mathbf{R}}$ is given by $L_{\mathbf{R}} \times P L(\Sigma) / M_{\mathbf{R}} \rightarrow \mathbf{R},(l, f) \mapsto \sum_{\bar{\mu} \in \mathcal{A}} l_{\mu} f(\mu)$. Let $\mathcal{M} \in L_{\mathbf{R}}$ be the cone generated by the primitive relations. Given $f \in \bar{K}\left(\mathbf{P}_{\Sigma}\right)$ (modulo linear functions) and a primitive collection $\mathcal{P}$, we have $\sum_{\mu \in \mathcal{P}} f(\mu) \geq f\left(\sum_{\mu \in \mathcal{P}} \mu\right)$. So $\sum_{\mu \in \mathcal{P}} f(\mu)-\sum_{\mu \in \mathcal{G}} c_{\mu} f(\mu) \geq 0$ or $\sum l(\mathcal{P})_{\mu} f(\mu) \geq 0$. So every $l(\mathcal{P})$ is in $\bar{K}\left(\mathbf{P}_{\Sigma}\right)^{\vee}$, i.e. $\mathcal{M} \subset \bar{K}\left(\mathbf{P}_{\Sigma}\right)^{\vee}$. We now show that $\mathcal{M}^{\vee} \subset \bar{K}\left(\mathbf{P}_{\Sigma}\right)$. Let $f \in \mathcal{M}^{\vee} \subset P L(\Sigma) / M_{\mathbf{R}}$. Then for all primitive collections $\mathcal{P}, \sum_{\bar{\mu} \in \mathcal{A}} l(\mathcal{P})_{\mu} f(\mu) \geq 0$, which is equivalent, once again, to $\sum_{\mu \in \mathcal{P}} f(\mu) \geq f\left(\sum_{\mu \in \mathcal{P}} \mu\right)$. Thus $f \in \bar{K}\left(\mathbf{P}_{\Sigma}\right)$.

Corollary 4.9. If $\tau$ is a cone contained in $\bar{K}\left(\mathbf{P}_{\Sigma}\right)$, then $\tau^{\vee}$ contains all the primitive relations.

We say that the fan $\Sigma$ has property $(*)$ if it satisfies:

$$
\Sigma(1) \subset \partial \operatorname{conv}(\Sigma(1)) . \quad(*)
$$

This just means that the primitive generators of the one-dimensional cones in the fan $\Sigma$ lie on the faces of a convex polytope. Note that each $(i+1)$-cone $\sigma \in \Sigma$ contains a canonical $i$-simplex $s_{\sigma}$ whose vertices are the primitive generators of $\sigma$. Property $(*)$ is also equivalent to the property that each $s_{\sigma}$ lie on a boundary face of $\operatorname{conv}(\Sigma(1))$.

Theorem 4.10. The following are equivalent:

(i) The first Chern class $c_{1}\left(\mathbf{P}_{\Sigma}\right) \in \bar{K}\left(\mathbf{P}_{\Sigma}\right)$.

(ii) Every primitive relation has $l(\mathcal{P})_{0} \leq 0$.

(iii) The fan $\Sigma$ has property $(*)$.

Proof. We first show that (i) $\Leftrightarrow$ (ii). Recall that $c_{1}\left(\mathbf{P}_{\Sigma}\right)$ is represented by the piecewise linear function $f:=\alpha_{\Sigma}$ with $f(\mu)=1$ for all $\mu \in \Sigma(1)$. By the above characterization of the Kähler cone, $c_{1}\left(\mathbf{P}_{\Sigma}\right) \in \bar{K}\left(\mathbf{P}_{\Sigma}\right)$ iff for every primitive collection $\mathcal{P}$ we have

$$
\sum_{\mu \in \mathcal{P}} f(\mu) \geq f\left(\sum_{\mu \in \mathcal{P}} \mu\right) .
$$

The last condition is equivalent to $|\mathcal{P}| \geq f\left(\sum_{\mu \in \mathcal{P}} \mu\right)=f\left(\sum_{\mu \in \mathcal{G}} c_{\mu} \mu\right)=\sum_{\mu \in \mathcal{G}} c_{\mu}$. The last equality uses the linearity of $f$ in $\operatorname{cone}(\mathcal{G})$. The inequality is in turn equivalent to $l(\mathcal{P})_{0}=\sum_{\mu \in \mathcal{G}} c_{\mu}-|\mathcal{P}| \leq 0$.

We now show (i) $\Leftrightarrow$ (iii). Consider the constant function which takes value 1 on $N_{\mathbf{R}}$. Its graph in $\bar{N}_{\mathbf{R}}$ is the hyperplane $1 \times N_{\mathbf{R}}$. The fan $\Sigma$ which covers $N_{\mathbf{R}}$ can be translated vertically to $\bar{\Sigma}$ which now covers $1 \times N_{\mathbf{R}}$. We denote by $s_{\bar{\sigma}}$ the translate of the simplex $s_{\sigma}$. Now we pull the apex $\bar{\mu}_{0}=1 \times \overrightarrow{0}$ down to the origin $0 \times \overrightarrow{0}$ piecewise linearly while holding each $s_{\bar{\sigma}}$ fixed. The resulting piecewise linear hypersurface in $\bar{N}_{\mathbf{R}}$ is the graph of the function $f$ representing $c_{1}\left(\mathbf{P}_{\Sigma}\right)$. It is clear that $f$ is a convex function iff $\bar{\Sigma}(1)$ lies on the boundary of the convex polytope conv $\bar{\Sigma}(1)$ in the hyperplane $1 \times N_{\mathbf{R}}$. Here $\bar{\Sigma}(1)$ is a translate of $\Sigma(1)$. So $\bar{\Sigma}(1)$ lies on the boundary of conv $\bar{\Sigma}(1)$ iff $\Sigma$ has property $(*)$.

This completes the proof. 


\section{Compactification And EXistence of MAXimal DEGEnERACY POINTS}

Now we consider a compactification of $\operatorname{Hom}\left(L, \mathbf{C}^{\times}\right)$in Proposition 3.4 using the two complete fans $S \Sigma$ and $G \Sigma$ in $L_{\mathbf{R}}^{*}=\operatorname{Hom}(L, \mathbf{Z}) \otimes \mathbf{R}$. Under our assumption that $\Sigma$ is a regular fan, we have the following exact sequence:

$$
0 \rightarrow L \rightarrow \mathbf{Z}^{\mathcal{A}} \rightarrow \bar{N} \rightarrow 0
$$

where $\mathbf{Z}^{\mathcal{A}} \rightarrow \bar{N}$ is given by $l \mapsto \sum_{\bar{\mu} \in \mathcal{A}} l_{\mu} \bar{\mu}$. This is onto because $\Sigma(1)$ generates the lattice $N$ since $\Sigma$ is regular. Dualizing the map $\mathbf{Z}^{\mathcal{A}} \rightarrow \bar{N}$, we get $\bar{M} \rightarrow \mathbf{Z}^{\mathcal{A}}$, $\bar{u} \mapsto \sum\langle\bar{u}, \bar{\mu}\rangle e_{\bar{\mu}}$, where the $e_{\bar{\mu}}$ is the standard basis of $\mathbf{Z}^{\mathcal{A}}$. Then we have the isomorphism

$$
L^{*} \simeq \mathbf{Z}^{\mathcal{A}} / \bar{M}
$$

With this integral structure, both $S \Sigma$ and $G \Sigma$ in $L^{*}$ consist of rational polyhedral cones and thus we can consider the toric varieties $\mathbf{P}_{S \Sigma}$ and $\mathbf{P}_{G \Sigma}$. According to Theorem 4.2, $\mathbf{P}_{G \Sigma}$ is a partial resolution of $\mathbf{P}_{S \Sigma}$ which is typically singular.

Problem 5.1 (Existence). Given an equivariant resolution $\phi: \mathbf{P}_{G \Sigma^{\prime}} \rightarrow \mathbf{P}_{G \Sigma}$, when does $\mathbf{P}_{G \Sigma^{\prime}}$ admit a maximal degeneracy point?

We will prove the following:

Theorem 5.2. If the regular fan $\Sigma$ has the property $(*)$ and the (Kähler) cone $K\left(\mathbf{P}_{\Sigma}\right)$ is a large cone in $L_{\mathbf{R}}^{*}$, then for every equivariant resolution $\phi: \mathbf{P}_{G \Sigma^{\prime}} \rightarrow \mathbf{P}_{G \Sigma}$, there is at least one maximal degeneracy point in $\mathbf{P}_{G \Sigma^{\prime}}$.

Remark 5.3. As we have proved, the combinatorial conditions in the hypotheses of the theorem are equivalent to the fact that the toric variety $\mathbf{P}_{\Sigma}$ is smooth, projective, and has $c_{1}\left(\mathbf{P}_{\Sigma}\right) \in \bar{K}\left(\mathbf{P}_{\Sigma}\right)$.

5.1. Indicial ideals. Let $\tau$ be a regular large cone in the space $L_{\mathbf{R}}^{*}$. Then it determines a unique $\mathbf{Z}$-basis $\left\{l^{(1)}, \ldots, l^{(p-n)}\right\}$ of $L$ in $\tau^{\vee} \cap L$, and hence a set of canonical coordinates $x_{\tau}^{(k)}$ of the affine variety $U_{\tau}=\operatorname{Hom}\left(\tau^{\vee} \cap L, \mathbf{C}\right)$. Thus by Proposition 3.4, we have

Proposition 5.4. For any regular large cone $\tau$ in $L_{\mathbf{R}}^{*}$, we have an embedding $\left(\mathbf{C}^{\times}\right)^{\mathcal{A}} /\left(\mathbf{C}^{\times} \times T_{M}\right) \rightarrow \operatorname{Hom}\left(\tau^{\vee} \cap L, \mathbf{C}\right)$, given by $a \mapsto x_{\tau}$ where $x_{\tau}^{(k)}=(-1)^{l_{0}^{(k)}} a^{l^{(k)}}$.

Recall that the interior of a large cone $\tau$ in the Gröbner fan $G \Sigma$ is an equivalence class of term order $\omega \in \mathbf{R}^{p+1}=\mathbf{R}^{\mathcal{A}}$ for the toric ideal $\mathcal{I}_{\mathcal{A}}$. We identify $\mathcal{I}_{\mathcal{A}}$ in $\mathbf{C}\left[y_{0}, \ldots, y_{p}\right]$ with the ideal generated by $\left\{\square_{l}\right\}_{l \in L}$ in $\mathbf{C}\left[\frac{\partial}{\partial a_{0}}, \ldots, \frac{\partial}{\partial a_{p}}\right]$. Let $B_{\omega}$ be a minimal Gröbner basis (see [24]) for the term order $\omega$. Then $B_{\omega}$ is a finite set of $\square_{l}$ with $\omega \cdot l \geq 0$ for all $\omega \in \tau$. Moreover, the leading term relative to $\omega$ is $L T_{\omega} \square_{l}=\left(\frac{\partial}{\partial a}\right)^{l^{+}}$. Now

$$
a^{l^{+}} \square_{l}=a^{l^{+}}\left(\frac{\partial}{\partial a}\right)^{l^{+}}-a^{l^{+}-l^{-}} a^{l^{-}}\left(\frac{\partial}{\partial a}\right)^{l^{-}} .
$$

Since $\omega \cdot\left(l^{+}-l^{-}\right) \geq 0$ for all $\omega \in \tau$, we have $l=l^{+}-l^{-} \in \tau^{\vee} \cap L$. Since $\tau^{\vee} \cap L$ is generated by $\left\{l^{(1)}, \ldots, l^{(p-n)}\right\}$, it follows from Proposition 5.4 that $a^{l^{+}-l^{-}}$in the second term can be expressed as a monomial of $\left\{x_{\tau}^{(k)}\right\}$ which vanishes when $x_{\tau}^{(k)} \rightarrow 0$. The first term in (5.3) is 'homogeneous' and can be written as a constant 
coefficient polynomial $I_{l}\left(\theta_{a_{0}}, \ldots, \theta_{a_{p}}\right)$ in the $\log$ derivatives $\theta_{a_{i}}=a_{i} \frac{\partial}{\partial a_{i}}$. Thus the leading term of $a^{-\gamma} a^{l^{+}} \square_{l} a^{\gamma}(1+O(x))$ is obviously $I_{l}(\gamma)$.

With the above motivation we define for any $l \in L$

$$
I_{l}(\gamma):=a^{-\gamma} a^{l^{+}}\left(\frac{\partial}{\partial a}\right)^{l^{+}} a^{\gamma} \in \mathbf{C}[\gamma]:=\mathbf{C}\left[\gamma_{0}, \ldots, \gamma_{p}\right] .
$$

Definition 5.5. For a cone $\tau \subset L_{\mathbf{R}}^{*}$ and an exponent $\beta=-1 \times \overrightarrow{0} \in \bar{N}_{\mathbf{R}}$, the indicial ideal $\operatorname{Ind}(\tau, \beta)$ is the ideal in $\mathbf{C}[\gamma]$ generated by

$$
I_{l}(\gamma) \text { with } l \in \tau^{\vee} \cap L, l \neq 0, \quad \sum\langle u, \bar{\mu}\rangle \gamma_{\mu}-\langle u, \beta\rangle \text { with } u \in \bar{M} .
$$

Note that the second set of relations is equivalent to $\sum_{\bar{\mu} \in \mathcal{A}} \bar{\mu} \gamma_{\mu}-\beta$. Note also that we make no assumption on the cone $\tau$. In practice, one can sometimes reduce the problem of finding the indices (i.e. the zero locus of the indicial ideal) by using a Gröbner basis of the toric ideal.

Proposition 5.6. Let $\tau$ be a large cone in the Gröbner fan, and let $B=$ $\left\{\square_{l^{(1)}}, \ldots, \square_{l^{(g)}}\right\}$ be a minimal Gröbner basis of $\mathcal{I}_{\mathcal{A}}$ for the class of term orders $\omega \in \tau$. Then the ideal

$$
\mathcal{J}_{B}:=\left\langle I_{l^{(i)}}(\gamma), i=1, \ldots, g ; \sum\langle u, \bar{\mu}\rangle \gamma_{\mu}-\langle u, \beta\rangle\right\rangle
$$

has the same zero locus as does $\operatorname{Ind}(\tau, \beta)$.

Proof. By definition, for each $i$, we have $L T_{\omega} \square_{l^{(i)}}=\left(\frac{\partial}{\partial a}\right)^{l^{(i)+}}$ and $\left\langle\omega, l^{(i)}\right\rangle>0$ for a term order $\omega \in \tau$. Thus $l^{(i)} \in \tau^{\vee} \cap L$. In particular, $\mathcal{J}_{B} \subset \operatorname{Ind}(\tau, \beta)$.

Suppose $\mathcal{J}_{B}$ vanishes at $t=\left(t_{0}, \ldots, t_{p}\right) \in \mathbf{C}^{p+1}$. We must show that so does $\operatorname{Ind}(\tau, \beta)$. It is enough to check that $I_{l}(t)=0 \forall l \in \tau^{\vee} \cap L \backslash(0)$. Let us fix $l$. Then any interior $\omega \in \tau$ has $\langle\omega, l\rangle>0$. Since $B$ is a Gröbner basis, we have $L T_{\omega} \square_{l}=\sum b_{i} L T_{\omega} \square_{l^{(i)}}$ for some differential operators $b_{i}$. This gives $\left(\frac{\partial}{\partial a}\right)^{l^{+}} a^{t}=$ $\sum b_{i}\left(\frac{\partial}{\partial a}\right)^{l^{(i)+}} a^{t}=0$. This implies that $I_{l}(t)=0$.

\subsection{Finite codimension theorem.}

Theorem 5.7. Suppose the regular fan $\Sigma$ has the property (*). If $\tau$ is any cone contained in $\bar{K}\left(\mathbf{P}_{\Sigma}\right)$, then there is a canonical onto ring homomorphism $A\left(\mathbf{P}_{\Sigma}\right) \otimes$ $\mathbf{C} \rightarrow \mathbf{C}[\gamma] / \operatorname{Ind}(\tau, \beta)$ with $D_{i} \mapsto \gamma_{i}$.

Proof. By Theorem 4.10, we have $l(\mathcal{P})_{0} \leq 0$ for each primitive collection $\mathcal{P}$. By Proposition 4.7, it follows that $l(\mathcal{P})_{0}^{+}=0$ and that $l(\mathcal{P})_{i}^{+}=1$ if $\mu_{i} \in \mathcal{P}$, and 0 otherwise. Thus we have $I_{l(\mathcal{P})}(\gamma)=a^{-\gamma} a^{l(\mathcal{P})^{+}}\left(\frac{\partial}{\partial a}\right)^{l(\mathcal{P})^{+}} a^{\gamma}=\gamma^{l(\mathcal{P})^{+}}$. As remarked earlier the elements $I_{l(\mathcal{P})}(D)=D^{l(\mathcal{P})^{+}}$, as $\mathcal{P}$ ranges over the primitive collections, generate precisely the Stanley-Reisner ideal of the fan $\Sigma$.

Consider the isomorphism $\mathbf{C}\left[\gamma_{0}, \ldots, \gamma_{p}\right] /\left\langle\sum \gamma_{i}+1\right\rangle \rightarrow \mathbf{C}\left[D_{1}, \ldots, D_{p}\right], \gamma_{i} \mapsto D_{i}$ for $i \neq 0, \gamma_{0} \mapsto-1-D_{1}-\cdots-D_{p}$. For $\beta=-1 \times \overrightarrow{0}$, it is clear that under this isomorphism we have

$$
\begin{aligned}
\left\langle\sum\left\langle u, \bar{\mu}_{i}\right\rangle \gamma_{i}-\langle u, \beta\rangle, u \in \bar{M}\right\rangle & =\left\langle\sum \gamma_{i}+1, \sum\left\langle u, \mu_{i}\right\rangle \gamma_{i}, u \in M\right\rangle \\
& \rightarrow\left\langle\sum\left\langle u, \mu_{i}\right\rangle D_{i}, u \in M\right\rangle .
\end{aligned}
$$


Comparing with the generators (i) of the Chow ideal, we get an isomorphism

$$
\mathbf{C}[\gamma] /\left\langle I_{l(\mathcal{P})}(\gamma), \mathcal{P} \text { primitive; } \sum\left\langle u, \bar{\mu}_{i}\right\rangle \gamma_{i}-\langle u, \beta\rangle, u \in \bar{M}\right\rangle \rightarrow A\left(\mathbf{P}_{\Sigma}\right) \otimes \mathbf{C} .
$$

By Definition 5.5 and Corollary 4.9, $\operatorname{Ind}(\tau, \beta)$ contains $I_{l(\mathcal{P})}(\gamma)$ for all primitive collections $\mathcal{P}$, and all $\sum\left\langle u, \bar{\mu}_{i}\right\rangle \gamma_{i}-\langle u, \beta\rangle, u \in M$. Thus inverting the above isomorphism, we get an onto homomorphism $A\left(\mathbf{P}_{\Sigma}\right) \otimes \mathbf{C} \rightarrow \mathbf{C}[\gamma] / \operatorname{Ind}(\tau, \beta)$.

Corollary 5.8. Under the hypotheses of Theorem 5.7, $\operatorname{Ind}(\tau, \beta)$ has finite codimension in $\mathbf{C}[\gamma]$.

Proof. This follows from the fact that $A\left(\mathbf{P}_{\Sigma}\right) \otimes \mathbf{C}$ is finite dimensional.

Corollary 5.9. Under the hypotheses of Theorem 5.7, the zero locus of the ideal $\left\langle I_{l(\mathcal{P})}(\gamma), \mathcal{P}\right.$ primitive; $\left.\sum\left\langle u, \bar{\mu}_{i}\right\rangle \gamma_{i}-\langle u, \beta\rangle, u \in \bar{M}\right\rangle$ has exactly one point $\gamma=$ $(-1,0, \ldots, 0) \in \mathbf{R}^{p+1}$.

Proof. As pointed out in the proof of the theorem, the relations in this ideal are

$$
\begin{aligned}
I_{l(\mathcal{P})}(\gamma)=0 & \text { with } \mathcal{P} \text { primitive }, \\
\sum\left\langle u, \mu_{i}\right\rangle \gamma_{i}=0 & \text { with } u \in M, \\
\sum \gamma_{i}+1=0 . &
\end{aligned}
$$

The first two sets of relations are identical to those of the Chow ideal. Since the Chow ideal is homogeneous and has finite codimension, the first two sets of relations above give $\gamma_{1}=\cdots=\gamma_{p}=0$. It follows from the third relation that $\gamma_{0}=-1$.

Corollary 5.10. Under the hypotheses of Theorem 5.7, the zero locus of the ideal $\operatorname{Ind}(\tau, \beta)$ has at most the one point $\gamma=(-1,0, \ldots, 0)$.

Proof. This follows from Theorem 5.7 and the preceding corollary.

Lemma 5.11. Let $S$ be a nonempty set of integral points in a large regular cone $C$ in $L_{\mathbf{R}}^{*}$. Then there exists a strongly convex regular cone $C^{\prime}$ containing both $C$ and the set $S-\delta$ for some $\delta \in S$.

Proof. Without loss of generality, we identify $C$ with the cone $\mathbf{R}_{\geq 0}^{p-n}$. It is enough to find a strongly convex cone $C^{\prime}$ (not necessary regular) with the above inclusion property, for every such cone is contained in a strongly convex regular cone. Now if $S$ contains the origin, then we set $C^{\prime}=C$. Otherwise we proceed as follows. Given $x \in C, v \in \operatorname{Int}\left(C^{\vee}\right)$, let $H_{v}(x)$ be the hyperplane in $\mathbf{R}^{p-n}$ containing $x$ and normal to $v$. We fix $x, v$ and let $t$ be the minimal positive number such that $H_{v}(t x)$ meets $S$. By perturbing $v$, if necessary, we may assume that $H_{v}(t x)$ meets $S$ at exactly one point $\delta$. In particular, we have $\langle v, s-\delta\rangle>0$ for all $s \in S \backslash\{\delta\}$. Performing a parallel translation of the hyperplane to $H_{v}(0)$ we see that the points of both $C$ and $S-\delta$, except the origin, all lie in the same open half-space bounded by $H_{v}(0)$. It follows that the normal cone to $C \cup(S-\delta)$ at 0 is strongly convex, and obviously has the desired inclusion property.

Corollary 5.12. Under the hypotheses of Theorem 5.7, if $\tau$ is a regular large cone in $L_{\mathbf{R}}^{*}$, then the $G K Z$ system has at most one power series solution of the form $a^{\gamma}(1+g(x))$ where $g(0)=0$. (The $x$ are the canonical coordinates of the affine variety $U_{\tau}=\operatorname{Hom}\left(\tau^{\vee} \cap L, \mathbf{C}\right)$.) Moreover, if this is a solution, then $\gamma=(-1,0, \ldots, 0)$. 
Proof. The second statement follows form the preceding corollary. For the first statement we show that any solution of the form $a^{\gamma} f(x)$ with $f(0)=0$ is identically zero. Suppose not. Then we can write $f(x)=\sum_{l \in \tau^{\vee}} \lambda_{l} a^{l}$ with $\lambda_{l}$ nonzero for some l. Let $S:=\left\{l \in \tau^{\vee} \mid \lambda_{l} \neq 0\right\}$. Since $f$ has no constant term, $S$ does not contain the origin. By the preceding lemma, we have a strongly convex regular cone $C^{\prime}$ containing both $\tau^{\vee}$ and $S-\delta$ for some $\delta \in S$. Now put $\lambda_{l} \equiv 0$ for $l$ outside $\tau^{\vee}$. We have

$$
a^{\gamma} f(x)=a^{\gamma} \sum_{l \in S} \lambda_{l} a^{l}=a^{\gamma+\delta} \sum_{l \in C^{\prime}} \lambda_{l+\delta} a^{l} .
$$

But the last sum can be written as $\lambda_{\delta} a^{\gamma+\delta}\left(1+g\left(x^{\prime}\right)\right)$ where $g(0)=0$, and the $x^{\prime}$ are the canonical coordinates of $U_{\sigma}\left(\sigma=C^{\prime \vee}\right)$. By the preceding corollary applied to $\sigma \subseteq \tau$, we have $\gamma+\delta=\gamma$, which means that $0=\delta \in S$. This contradicts the fact that $S$ does not contain the origin.

The ideal $\operatorname{Ind}(\tau, \beta)$ can be all of $\mathbf{C}[\gamma]$ in which case its zero locus is empty. For example, if $\tau=(0)$ and $l_{1} \mu_{1}+\cdots+l_{p} \mu_{p}=0$ for some nonpositive integers $l_{1}, \ldots, l_{p}$, not all zero, then $l=\left(l_{0}:=-l_{1}-\cdots-l_{p}, l_{1}, \ldots, l_{p}\right) \in L$ and we have $I_{l}(-1,0, \ldots, 0) \neq 0$.

Corollary 5.13. Under the hypotheses of Theorem 5.7, the zero locus of the ideal $\operatorname{Ind}(\tau, \beta)$ has exactly the one point $\gamma=(-1,0, \ldots, 0)$ iff every nonzero $l \in \tau^{\vee} \cap L$ has $l_{i}>0$ for some $i>0$.

Proof. It is obvious that the point $\gamma=(-1,0, \ldots, 0)$ satisfies the second and third relations in (5.9). So we need only to consider the conditions $I_{l}(-1,0, \ldots, 0)=0$ for all $l \in \tau^{\vee} \cap L, l \neq 0$. By definition, $I_{l}(-1,0, \ldots, 0)=a_{0} a^{l^{+}}\left(\frac{\partial}{\partial a}\right)^{l^{+}} a_{0}^{-1}$. It is obvious that this is zero iff $l_{i}>0$ for some $i>0$.

5.3. Proof of the main Theorem 5.2. In this section, we will assume that the fan $\Sigma$ is regular and has property $(*)$, and that $\bar{K}\left(\mathbf{P}_{\Sigma}\right)$ is a large cone. It is obvious that the fan endows the polytope $\operatorname{conv}(\mathcal{A})$ with a natural triangulation $T^{0}$ whose $n$-simplices correspond 1-1 to the large cones in $\Sigma$. We will call this a maximal triangulation $T^{0}$. Then under the identifications $\mathbf{R}^{p+1} / \bar{M}_{\mathbf{R}}=\mathbf{R}^{p} / M_{\mathbf{R}}=L_{\mathbf{R}}^{*}$, we have $\mathcal{C}\left(T^{0}\right)=\bar{K}\left(\mathbf{P}_{\Sigma}\right)$. Since $\bar{K}\left(\mathbf{P}_{\Sigma}\right)$ is a large cone, the triangulation $T^{0}$ is regular.

We can regard the cone $\bar{K}\left(\mathbf{P}_{\Sigma}\right)$ together with all its faces as a fan, which we denote $K$. Corresponding to this fan is an affine toric variety

$$
\mathbf{P}_{K}=\operatorname{Hom}\left(\bar{K}\left(\mathbf{P}_{\Sigma}\right)^{\vee} \cap L, \mathbf{C}\right) .
$$

It is of course canonically a toric subvariety of $\mathbf{P}_{S \Sigma}$. In $\mathbf{P}_{K}$ lies a unique torus fixed point $p_{K}$. In general $p_{K}$ is singular. Let $\mathbf{P}_{K^{\prime}} \rightarrow \mathbf{P}_{K}$ be any equivariant resolution (thus $K^{\prime}$ is a regular refinement of $K$ ). The fiber over $p_{K}$ is a union of torus orbits. From standard toric geometry, we get

Lemma 5.14. Given $\mathbf{P}_{K^{\prime}} \rightarrow \mathbf{P}_{K}$, the torus fixed points in the fiber over $p_{K}$ correspond 1-1 with the large cones $\tau \in K^{\prime}$.

We denote by $p_{\tau}$ the fixed point corresponding to $\tau$. As pointed out earlier, the period integrals $\bar{\Pi}_{\alpha}$ are local sections of a sheaf over the open variety $S_{\mathcal{A}}$; and the latter is canonically an open subvariety in every toric variety $\mathbf{P}_{F}$ corresponding to any rational fan $F$ defined in $L_{\mathbf{R}}^{*}$. In particular, for the fan $K^{\prime}$, we can ask for the behavior of the period integrals near $p_{\tau} \in \mathbf{P}_{K^{\prime}}-S_{\mathcal{A}}$. 
Recall that we have an explicit formula for a period integral $\bar{\Pi}_{\alpha_{0}}$ given by Proposition 3.1. Near $p_{\tau}$, we can write this period in the canonical coordinates $x=x_{\tau}$ using Proposition 5.4.

Proposition 5.15. Fix a large cone $\tau \in K^{\prime}$ and let $l^{(1)}, \ldots, l^{(p-n)} \in L$ be the canonical generators of $\tau^{\vee}$. Near $p_{\tau}$ in the canonical coordinates $x=x_{\tau}$, the period integral $\bar{\Pi}_{\alpha_{0}}$ can be be written as

$$
\bar{\Pi}_{\alpha_{0}}=\sum_{m_{1}, \ldots, m_{p-n} \geq 0, \sum m_{k} l_{0}^{(k)} \leq 0} \frac{\Gamma\left(-\sum m_{k} l_{0}^{(k)}+1\right)}{\prod_{1 \leq i \leq p} \Gamma\left(\sum m_{k} l_{i}^{(k)}+1\right)} x^{m} .
$$

In particular, it extends holomorphically across $p_{\tau}$.

Proof. Since $\tau \subset \bar{K}\left(\mathbf{P}_{\Sigma}\right)=\mathcal{C}\left(T^{0}\right)$, we have $\tau^{\vee} \supset \mathcal{C}\left(T^{0}\right)^{\vee}=\overline{\mathcal{C}}^{\prime}\left(T^{0}\right)^{\vee}$. Thus it follows from Proposition 4.1 that $\tau^{\vee}$ contains the $K(I):=\left\{x \in L_{\mathbf{R}} \mid x_{i} \geq 0, i \notin I\right\}$ for all $I \in T^{0}$. Since $T^{0}$ is the triangulation of $\operatorname{conv}(\mathcal{A})$ induced by the fan $\Sigma$, every $n$-simplex of $T^{0}$ has the point $\bar{\mu}_{0}=1 \times \overrightarrow{0}$ as a vertex, i.e., every basis $I \in T^{0}$ contains this point. It follows that for every tuple $\left(l_{1}, \ldots, l_{p}\right)$ which we sum over in Proposition 3.1 , if we set $l_{0}=-l_{1}-\cdots-l_{p}$, then we get $l:=\left(l_{0}, \ldots, l_{p}\right) \in K(I) \subset$ $\tau^{\vee}$. So there exist unique integers $m_{1}, \ldots, m_{p-n} \geq 0$ such that $l_{i}=\sum m_{k} l_{i}^{(k)}$ for all $i$. Thus $(-1)^{l_{1}+\cdots+l_{p}} \frac{\left(l_{1}+\cdots+l_{p}\right) !}{l_{1} ! \cdots l_{p} !} \frac{a_{1}^{l_{1} \cdots a_{p}}}{a_{0}^{l_{1}+\cdots+l_{p}}}$ coincides with $\frac{\Gamma\left(-\sum m_{k} l_{0}^{(k)}+1\right)}{\prod_{1<i<p} \Gamma\left(\sum m_{k} l_{i}^{(k)}+1\right)} x^{m}$. Conversely, given a nonnegative tuple $\left(m_{1}, \ldots, m_{p-n}\right)$ with $\sum m_{k} l_{0}^{(k)} \leq 0$, the last term is either zero (when some $\Gamma\left(\sum m_{k} l_{i}^{(k)}+1\right.$ ) is finite), or it coincides with some $(-1)^{l_{1}+\cdots+l_{p}} \frac{\left(l_{1}+\cdots+l_{p}\right) !}{l_{1} ! \cdots l_{p} !} \frac{a_{1}^{l_{1}} \cdots a_{p}^{l_{p}}}{a_{0}^{l_{1}+\cdots+l_{p}}}$ when nonzero.

Thus, on the one hand, we have one period integral which extends across $p_{\tau}$. On the other hand, there cannot be any other period integrals with this property by Corollary 5.12. So we conclude that

Theorem 5.16. For any equivariant resolution $\mathbf{P}_{K^{\prime}} \rightarrow \mathbf{P}_{K}$, near a given torus fixed point in $\mathbf{P}_{K^{\prime}}, \bar{\Pi}_{\alpha_{0}}$ extends holomorphically across that point and is the only period integral which does so.

Corollary 5.17. For any equivariant resolution $\phi: \mathbf{P}_{S \Sigma^{\prime}} \rightarrow \mathbf{P}_{S \Sigma}$ near a given torus fixed point in $\phi^{-1}\left(p_{K}\right), \bar{\Pi}_{\alpha_{0}}$ extends holomorphically across that point and is the only period integral which does so.

Proof. Since $\bar{K}\left(\mathbf{P}_{\Sigma}\right)$ is a large cone in the secondary fan $S \Sigma$, we can regard the fan $K$ (consisting of all the faces of $\bar{K}\left(\mathbf{P}_{\Sigma}\right)$ ) as a subfan of $S \Sigma$. The preimage $K^{\prime}:=\phi^{-1}(K) \subset S \Sigma^{\prime}$ is a regular fan refinement of $K$, and the induced map $\mathbf{P}_{K^{\prime}} \rightarrow \mathbf{P}_{K}$ is a restriction of $\phi: \mathbf{P}_{S \Sigma^{\prime}} \rightarrow \mathbf{P}_{S \Sigma}$. Applying the preceding theorem, we get the desired result.

Since the Gröbner fan $G \Sigma$ is a refinement of the secondary fan $S \Sigma$, any equivariant resolution $\mathbf{P}_{G \Sigma^{\prime}} \rightarrow \mathbf{P}_{G \Sigma}$ is also a resolution of $\mathbf{P}_{S \Sigma}$. Thus the preceding corollary applies. This proves our main Theorem 5.2.

Remark 5.18. Actually we have proved a stronger result than Theorem 5.2. Not only is $\bar{\Pi}_{\alpha_{0}}$ the only period integral that extends across a torus fixed point given above, but this is the only (up to a multiple) solution to the GKZ system which extends. 


\section{GRÖBNER BASES}

As we have seen, we can obtain rather detailed information about how period integrals, or more generally the solutions to the GKZ system, behave near a particular boundary point by studying a cone in the secondary fan. In the preceding discussion it was the Kähler cone and the corresponding torus fixed point which played an essential role.

There is in fact more to the story. We can actually reduce the GKZ system to a finite systems of PDEs in a canonical way. These finite systems correspond 1-1 to the Gröbner bases of the toric ideal. The Gröbner fan $G \Sigma$ turns out to be just the right set up for constructing these finite systems. One can then use them to study explicit solutions to the GKZ system near various boundary components in the parameter space. Near a maximal degeneracy point given above, the finite system turns out to have a uniform and simple description in terms of primitive collections of the fan $\Sigma$.

For generic values of the exponent $\beta$ (the so-called nonresonant cases), the theory of Gel'fand-Kapranov-Zelevinsky [12] gives a uniform description for the solutions to the GKZ system. However, for our special values of the exponent $\beta=-1 \times 0$ an analogous uniform description is not known. This exceptional case is especially important for applications in mirror symmetry. In this case, there are conjectural formulas for general solutions to the GKZ system ([16], [17]). Using our construction of finite PDE system, we can explicitly verify these solution formulas.

6.1. The maximal triangulation $T^{0}$. As in the previous section, we assume the fan $\Sigma$ is regular and has the property $(*)$. We also assume the cone $K\left(\mathbf{P}_{\Sigma}\right)$ is large. As we have seen at the beginning of Section 5.2, we can associate the fan $\Sigma$ to the maximal triangulation $T^{0}$ of $\mathcal{A}$. Then the cone $\mathcal{C}^{\prime}\left(T^{0}\right)$ in the secondary fan coincides with the closure $\bar{K}\left(\mathbf{P}_{\Sigma}\right)$.

Proposition 6.1. For any term order $\omega$ with $T_{\omega}=T^{0}$, we have $S R_{T^{0}}=L T_{\omega}\left(\mathcal{I}_{\mathcal{A}}\right)$, i.e., $L T_{\omega}\left(\mathcal{I}_{\mathcal{A}}\right)$ is radical.

Proof. By Theorem 4.10, $l(\mathcal{P})_{0} \leq 0$ for every primitive collection $\mathcal{P}$ of the fan $\Sigma$. Since $T_{\omega}=T^{0}$, we have $\omega \in \operatorname{Int} \mathcal{C}^{\prime}\left(T^{0}\right)$. Thus $\omega$ modulo $\bar{M}_{\mathbf{R}}$ is an element $f$ in the Kähler cone $K\left(\mathbf{P}_{\Sigma}\right) \subset P L(\Sigma) / M_{\mathbf{R}}$. This is the piecewise linear function given by $f\left(\mu_{i}\right)=\omega_{i}-\omega_{0}$. By Theorem 4.6, for every primitive collection $\mathbf{P}$ of $\Sigma$ we have

$$
\sum_{\mu \in \mathcal{P}} f(\mu)>f\left(\sum_{\mu \in \mathcal{P}} \mu\right) .
$$

If we write $\sum_{\mu \in \mathcal{P}} \mu=\sum_{\mu \in \mathcal{G}} c_{\mu} \mu$, where $\mathcal{G}$ is the generator set of the smallest cone in $\Sigma$ containing $\sum_{\mu \in \mathcal{P}} \mu$, then (6.1) reads $\langle\omega, l(\mathcal{P})\rangle>0$. This implies that $L T_{\omega}\left(y^{l(\mathcal{P})^{+}}-y^{l(\mathcal{P})^{-}}\right)=y^{l(\mathcal{P})^{+}}$. Since $l(\mathcal{P})_{0} \leq 0$, it follows that $l(\mathcal{P})_{i}^{+}=1$ if $\mu_{i} \in \mathcal{P}$ and 0 otherwise. Hence $y^{l(\mathcal{P})^{+}}=y_{\mathcal{P}}$. Since $S R_{T^{0}}=\left\langle y_{\mathcal{P}}\right| \mathcal{P}$ primitive $\rangle$, it follows that $S R_{T^{0}} \subset L T_{\omega}\left(\mathcal{I}_{\mathcal{A}}\right)$. By Theorem 4.2, we have the reverse inclusion.

Corollary 6.2. The cone $\bar{K}\left(\mathbf{P}_{\Sigma}\right)$ is in the Gröbner fan $G \Sigma$.

Proof. We must show that any two term orders $\omega, \omega^{\prime}$ in $K\left(\mathbf{P}_{\Sigma}\right)=\operatorname{Int} \mathcal{C}^{\prime}\left(T^{0}\right)$ are equivalent. Now their being in $\mathcal{C}^{\prime}\left(T^{0}\right)$ means that $T_{\omega}=T^{0}=T_{\omega^{\prime}}$. It follows from the preceding proposition that $L T_{\omega}\left(\mathcal{I}_{\mathcal{A}}\right)=L T_{\omega^{\prime}}\left(\mathcal{I}_{\mathcal{A}}\right)$. 
Corollary 6.3. The set $\left\{y^{l(\mathcal{P})^{+}}-y^{l(\mathcal{P})^{-}} \mid \mathcal{P}\right.$ primitive $\}$ is a minimal Gröbner basis of the toric ideal $\mathcal{I}_{\mathcal{A}}$ for a term order $\omega$ with $T_{\omega}=T^{0}$.

Proof. From the proof of the above proposition, we see that the leading terms $y_{\mathcal{P}}$ from the given set generate $L T_{\omega}\left(\mathcal{I}_{\mathcal{A}}\right)$, and so this set is a Gröbner basis. Moreover the leading terms have coefficient 1 , and $y_{\mathcal{P}}$ from one primitive collection $\mathcal{P}$ does not divide $y_{\mathcal{P}^{\prime}}$ from another $\mathcal{P}^{\prime}$ unless $\mathcal{P}=\mathcal{P}^{\prime}$.

According to Corollary 6.2, the preimage of the torus fixed point $p_{K} \in \mathbf{P}_{S \Sigma}$ under the blow-up $\mathbf{P}_{G \Sigma} \rightarrow \mathbf{P}_{S \Sigma}$ is a single point. We denote it as $p_{K}$ also.

Corollary 6.4. Let $\phi: \mathbf{P}_{G \Sigma^{\prime}} \rightarrow \mathbf{P}_{G \Sigma}$ be an equivariant resolution of the 'Gröbner variety'. Every torus fixed point in $\phi^{-1}\left(p_{K}\right)$ is a maximal degeneracy point.

Conjecture 6.5. Given $\phi: \mathbf{P}_{G \Sigma^{\prime}} \rightarrow \mathbf{P}_{G \Sigma}$ as above, the only maximal degeneracy points should be torus fixed points in $\phi^{-1}\left(p_{K}\right)$.

6.2. The GKZ system and primitive collections. If we identify $\mathbf{C}\left[y_{0}, \ldots, y_{p}\right]$ with the ring of differential operators $\mathbf{C}\left[\frac{\partial}{\partial a_{0}}, \ldots, \frac{\partial}{\partial a_{p}}\right]$ in an obvious way, then the toric ideal $\mathcal{I}_{\mathcal{A}}$ becomes precisely the ideal generated by the order $>1$ operators in the GKZ system. Thus a Gröbner basis [9] for the toric ideal allows us to reduce the GKZ system to a finite system in a canonical fashion.

For example, fix an equivariant resolution $\phi: \mathbf{P}_{G \Sigma^{\prime}} \rightarrow \mathbf{P}_{G \Sigma}$. We consider the GKZ system near the torus fixed point $p_{\tau} \in \phi^{-1}\left(p_{K}\right)$. Relative to the term orders $\omega$ with $T_{\omega}=T^{0}$, Corollary 6.3 reduces the GKZ system to the finite system of equations

$$
a^{-\gamma} \square_{l(\mathcal{P})} a^{\gamma} f(x)=0
$$

indexed by the primitive collections $\mathcal{P}$ of $\Sigma$. Here $\gamma$ satisfies $\sum \gamma_{\mu} \bar{\mu}=\beta$, and $x=x_{\tau}$ are the canonical coordinates of the affine subvariety $U_{\tau} \subset \mathbf{P}_{G \Sigma^{\prime}}$.

Near other torus fixed points, not necessarily in $\phi^{-1}\left(p_{K}\right)$, the GKZ system can also be reduced to a finite system using Gröbner bases of the toric ideal relative to other term orders. There is a degree bound for the binomials appearing in such Gröbner bases [23]. There is also a combinatorial description for the universal Gröbner basis. We have worked out some examples of this in [17].

6.3. Mirror symmetry. As we pointed out earlier, the general theory we have discussed arises naturally in mirror symmetry. Let us first consider the famous example studied by Greene-Plesser [14] and Candelas et al. [6]. Let $\nabla$ be the standard 4-simplex in $\mathbf{R}^{4}$ with vertices at the basis point $e_{1}, \ldots, e_{4}$ and at $-e_{1}-$ $\cdots-e_{4}$. Let $\Sigma$ be the fan consisting of cones over the faces of $\nabla$. Then the toric variety $\mathbf{P}_{\Sigma}$ coincides with the projective space $\mathbf{P}^{4}$. The set $\mathcal{A}:=\left\{1 \times \mu \mid \mu \in \nabla \cap \mathbf{Z}^{4}\right\}$ has 6 points. Let $\Delta$ be the polar dual of $\nabla$. The fan over the faces of $\Delta$ is not regular but admits a regular refinement, which we denote $\Phi$. Let $\mathcal{L}=K_{\mathbf{P}_{\Phi}}^{-1}$ be the anticanonical bundle of $\mathbf{P}_{\Phi}$. The space of sections $H^{0}(\mathcal{L})$ is isomorphic to $\mathbf{C}^{\mathcal{A}}$, and the 5-dimensional projective space $\mathbf{P} H^{0}(\mathcal{L})=\mathbf{P C}^{\mathcal{A}}$ parameterizes a family of Calabi-Yau hypersurfaces $Z_{a}$ in $\mathbf{P}_{\Phi}$. Here $Z_{a}, a \in \mathbf{P} \mathbf{C}^{\mathcal{A}}$, is the hypersurface given by the closure in $\mathbf{P}_{\Phi}$ of the zero locus of $f_{\mathcal{A}}(X, a)$. Then via the Poincaré residue map, the periods of the $Z_{a}$ can be written as period integrals

$$
a_{0} \int_{\alpha} \frac{1}{f_{\mathcal{A}}(X, a)} \prod \frac{d X_{i}}{X_{i}}
$$


The Gröbner variety $\mathbf{P}_{G \Sigma}$ in this case is $\mathbf{P}^{1}$. It is easy to show that with a suitable choice of coordinates, $x=0$ is the unique maximal degeneracy point in $\mathbf{P}^{1}$. A remarkable discovery in [6] is that a particular combination of the period integrals produces a generating function which predicts the number of rational curves in each degree in a generic quintic $Y$ in $\mathbf{P}_{\Sigma}=\mathbf{P}^{4}$. A quintic $Y$ in $\mathbf{P}^{4}$ and a Calabi-Yau hypersurface $Z$ in $\mathbf{P}_{\Phi}$ make what is known as a mirror pair, for the reason that their Hodge diamonds are mirror reflections of one another.

There is a straightforward generalization of the above example to $n \geq 4$. More generally Batyrev have given a construction of pairs of Calabi-Yau hypersurfaces from pairs of reflexive polytopes generalizing the pair $\nabla, \Delta$ above and all previously known examples. An important question arising from each such pair is the question of existence of maximal degeneracy points in the deformation space of the hypersurfaces. Our general theory for studying period integrals specializes to these cases, settles the question of existence of maximal degeneracy points, and has given a uniform construction of these points. The existence and construction of such points are crucial for the computation of (the analogues) of the Candelas et al. generating function. We have verified in [17] that our construction in all previously known plus some new examples, correctly predicts this generating function.

\section{REFERENCES}

1. V. Batyrev, J. Algebraic Geometry 3 (1994), 493-535. MR 95c:14046

2. __ Duke Math. J. 69 (1993), 349-409. MR 94m:14067

3. _ Quantum cohomology rings of toric manifolds, preprint 1993.

4. P. Berglund, S. Katz and A. Klemm, Nucl. Phys. B456 (1995), 153-204. CMP 96:07

5. L. Billera, P. Filliman and B. Sturmfels, Adv. in Math. 83 (1990), 155-179. MR 92d:52028

6. P. Candelas, X. de la Ossa, P. Green and L. Parks, Nucl. Phys. B359 (1991), 21-74. MR 93b:32029

7. P. Candelas, X. de la Ossa, A. Font, S. Katz and D. Morrison, Nucl. Phys. 416 (1994), 481-538. MR 95k:32020

8. P. Candelas, A. Font, S. Katz and D. Morrison, Nucl. Phys. B429 (1994), 626-674. MR 96g:32038

9. D. Cox, J. Little and D. O'Shea, Ideals, Varieties and Algorithms, UTM Springer-Verlag, 1992. MR 93j: 13031

10. A. Font, Nucl. Phys. B391 (1993), 358-388. MR 94d:32030

11. W. Fulton, An introduction to toric varieties, Princeton Univ. Press 1993. MR 94g:14028

12. I. M. Gel'fand, A. V. Zelevinsky and M. M. Kapranov, Funct. Anal. Appl. 23 (1989), 94-106. MR 90m:22025

13. _ Adv. Math. 84 (1990), 237-254, 255-271. MR 92a:14060; MR 92e:33015

14. B. Greene and M. Plesser, Nucl. Phys. B338 (1990), 15-37. MR 91h:32018

15. S. Hosono, A. Klemm, S. Theisen and S.-T. Yau, Commun. Math. Phys. 167 (1995), 301-350. MR 96a: 32044

16. , Nucl. Phys. B433 (1995), 501-552. MR 96d:32028

17. S. Hosono, B. Lian and S. T. Yau, GKZ-Generalized Hypergeometric Systems in Mirror Symmetry of Calabi-Yau Hypersurfaces, Harvard Univ. preprint, alg-geom/9511001, to appear in CMP 1996.

18. A. Klemm and S. Theisen, Nucl. Phys. B389 (1993), 153-180. MR 94d:32029

19. D. Morrison, Picard-Fuchs Equations and Mirror Maps for Hypersurfaces, in Essays on Mirror Manifolds, Ed. S.-T. Yau, International Press, 1992. MR 94b:32035

20. D. Mumford, J. Fogarty and F. Kirwan, Geometric Invariant Theory, 3rd Ed., SpringerVerlag, 1994. MR 95m:14012

21. T. Oda, Convex Bodies and Algebraic Geometry, Springer-Verlag, 1988. MR 88m:14038

22. T. Oda and H. S. Park, Tôhoku Math. J. 43 (1991), 375-399. MR 92d:14042

23. B. Sturmfels, Tôhoku Math. J. 43 (1991), 249-261. MR 92j:14067 
24. _ Gröbner Bases and Convex Polytopes, AMS University Lecture Series, Vol. 8, Providence, RI, 1995. CMP 96:05

25. _ Essays on Mirror Manifolds, Ed. S.-T. Yau, International Press, 1992. MR 94b:32001

ABSTRACT. Motivated by mirror symmetry, we study certain integral representations of solutions to the Gel'fand-Kapranov-Zelevinsky (GKZ) hypergeometric system. Some of these solutions arise as period integrals for Calabi-Yau manifolds in mirror symmetry. We prove that for a suitable compactification of the parameter space, there exist certain special boundary points, which we called maximal degeneracy points, at which all solutions but one become singular.

Department of Mathematics, Toyama University, Toyama 930, Japan

E-mail address: hosono@sci.toyama-u.ac.jp

Department of Mathematics, Brandeis University, Waltham, Massachusetts 02154

E-mail address: lian@max.math.brandeis.edu

Department of Mathematics, Harvard University, Cambridge, Massachusetts 02138 